\title{
Reduction of the transcription level of the mitochondrial genome in human glioblastoma
}

\author{
Vladimir Dmitrenko ${ }^{a}$, Katherina Shostak ${ }^{\mathrm{a}}$, Oxana Boyko ${ }^{\mathrm{a}}$, Olexiy Khomenko ${ }^{\mathrm{b}}$, \\ Vladimir Rozumenko ${ }^{\mathrm{b}}$, Tatiana Malisheva ${ }^{\mathrm{b}}$, Mikhail Shamayev ${ }^{\mathrm{b}}$, \\ Yuriy Zozulya ${ }^{\mathrm{b}}$, Vadym Kavsan ${ }^{\mathrm{a}, *}$
}

\author{
${ }^{a}$ Department of Molecular Biology, Institute of Molecular Biology and Genetics, 150 Zabolotnogo str., 03143 Kiev, Ukraine \\ ${ }^{\mathrm{b}}$ A.P. Romodanov Institute of Neurosurgery, 32 Manuilskogo str., 04050 Kiev, Ukraine
}

Received 26 April 2004; received in revised form 28 June 2004; accepted 1 July 2004

\begin{abstract}
Screening of human fetal brain cDNA library by glioblastoma (GB) and normal human brain total cDNA probes revealed 80 differentially hybridized clones. Hybridization of the DNA from selected clones and the same cDNA probes confirmed this difference for 38 clones, of which eight clones contained Alu-repeat inserts with increased levels in GB. Thirty clones contained cDNAs corresponding to mitochondrial genes for ATP synthase subunit 6 (ATP6), cytochrome $c$ oxidase subunit II (COXII), cytochrome $c$ oxidase subunit III (COXIII), NADH dehydrogenase subunit 1 (ND1), NADH dehydrogenase subunit 4 (ND4), and mitochondrial $12 \mathrm{~S}$ rRNA. The levels of all these mitochondrial transcripts were decreased in glioblastomas as compared to tumor-adjacent histologically normal brain. Earlier we found the same for cytochrome $c$ oxidase subunit I (COXI) Serial Analysis of Gene Expression (SAGE) showed lower content of the tags for all mitochondrial genes in GB SAGE libraries and together with our experimental data could serve as evidence of general inactivation of the mitochondrial genome in glioblastoma - the most malignant and abundant form of human brain tumor.
\end{abstract}

(C) 2004 Published by Elsevier Ireland Ltd.

Keywords: Astrocytic gliomas; Glioblastoma; Differential expression; Mitochondrial genes; Differential hybridization; Serial analysis of gene expression

\section{Introduction}

Malignant gliomas are very aggressive, highly invasive and neurologically destructive tumors of the central nervous system. In the most aggressive manifestation of glioma, glioblastoma (GB), median

\footnotetext{
* Corresponding author. Tel.: +380-44-266-3498; fax: +38044-266-0759.

E-mail address: kavsan@imbg.org.ua (V. Kavsan).
}

survival ranges from 9 to 12 months despite technical progress in neurosurgery, chemical, and radiation therapy. Although a comprehensive view of the genetic lesions encountered in malignant astrocytic gliomas has been compiled, present knowledge reflects only a fraction of the biological mechanisms assumed to initiate and promote astrocytoma formation. Changes in gene expression are important determinants of normal cellular physiology and, if disturbed, directly contribute to abnormal cellular physiology, including 
cancer. Characterization of genes either activated or repressed in GB will contribute to our understanding of the mechanisms underlying tumor initiation and progression. Knowledge of such mechanisms is likely to improve diagnostic and prognostic evaluation of astrocytic gliomas, as well as therapeutic approaches towards these tumors.

In this study, differential hybridization of human fetal brain cDNA library with total cDNA probes of GB and human normal brain was used for the identification of differentially expressed genes. Hybridization analysis revealed decreased expression in GB for seven mitochondrial $(\mathrm{mt})$ genes. Mitochondria are involved either directly or indirectly in many aspects of metabolism in cancer cells and notable differences in the bioenergetics and oxidative metabolism between normal and transformed cells have been discovered [1-4]. Differences between the mitochondria of cancer cells and those of normal cells result in the respiratory deficiencies common to rapidly growing cancer cells. The mutations were identified in different $\mathrm{mt}$ genes. The very high frequency of mtDNA mutations and the increase of mtDNA contents in pancreatic cancer cells led the authors to speculate that mtDNA may serve as a diagnostic tool [5]. It is not clear if the increase of mtDNA mass reflects an increased replication of mtDNA in response to defective mtDNA function due to extensive mutations. There is also abundant evidence for the alteration of the mitochondrial genome transcription in different cancer cells: in some tumors and tumor cell lines, transcription of mitochondrial genes has been shown to occur at a lower rate than in normal tissue, while in different other tumors mitochondrial genes express mostly at a higher level (for example, see reviews of Refs. [6-9]).

Our results extend previous data concerning the changes of mitochondrial genes expression in tumors and show general inactivation of the mitochondrial genome in glioblastoma, the last stage of astrocytic glioma progression.

\section{Materials and methods}

\subsection{Tumor and normal tissue samples}

Samples of astrocytic gliomas, WHO grade II and grade III, GBs and other types of brain tumors were obtained from the A P. Romodanov Institute of Neurosurgery (Kiev). Tumors were classified on the basis of review of haematoxylin and eosin stained sections of surgical specimens according to World Health Organization (WHO) criteria [10]. Surgical specimens of histologically normal brain tissue adjacent to tumors were used as a source of normal adult human brain RNA. Altogether, 41 gliomas and 3 non-glial tumors were studied. Tumors included 14 GBs (astrocytoma WHO grade IV), 15 anaplastic astrocytomas (astrocytoma WHO grade III), 9 astrocytomas (astrocytoma WHO grade II), two oligoastrocytoma (WHO grade II), one anaplastic oligoastrocytoma (WHO grade III), one epidermoid, one angiosarcoma, and one lymphoma.

\subsection{Differential hybridization of cDNA library}

Human fetal brain cDNA library (library No 415, 25 week old) was received from the Resource Center/ Primary DataBase (RZPD) of the German Human Genome Project (Max Planck Institute of Molecular Genetics, Berlin) The library consists of two filters with double spotted 27648 cDNA clones on each filter. Total cDNA probes for differential hybridization were synthesized from total RNA isolated from surgical specimens of primary GB and normal adult human brain. The conditions of oligo(dT)-primed cDNA synthesis were as described previously [11], but the concentration of dCTP was reduced to $25 \mu \mathrm{M}$ and $150-200 \mu \mathrm{Ci}$ of $\left[\alpha-{ }^{32} \mathrm{P}\right] \mathrm{dCTP}$ to obtain cDNA probes with high specific activity [12]. cDNA filter arrays were sequentially hybridized with tumor and normal brain cDNA probes and the coordinates of cDNA clones with altered hybridization signals between tumor and control cDNAs were determined on the gridded arrays. The clones were requested from RZPD (http://www. rzpd.de) and repeated differential hybridization of these clones was done.

\subsection{RNA isolation and Northern blot analysis}

Total RNA was isolated from frozen tissues according to Chomczynsky and Sacchi [13] RNA (10 $\mu \mathrm{g}$ per lane) was electrophoretically separated in a $1.5 \%$ agarose gel containing $2.2 \mathrm{M}$ formaldehyde and then transferred to a Hybond-N nylon membrane (Amersham Pharmacia Biotech, Austria). ${ }^{32} \mathrm{P}$-labelled probes of mitochondrial cDNAs were produced with 
RediPrime II kit (Amersham Pharmacia Biotech). The membranes were hybridized with ${ }^{32} \mathrm{P}$-labelled mitochondrial cDNA probes in $50 \%$ formamide, $5 \times \mathrm{SSC}$, $5 \times$ Denhardt's solution, $0.1 \%$ SDS and $100 \mu \mathrm{g} / \mathrm{ml}$ salmon sperm DNA at $42{ }^{\circ} \mathrm{C}$ overnight. Extensive washing was performed: twice with $2 \times \mathrm{SSC}, 0.1 \%$ SDS for $15 \mathrm{~min}$ at room temperature; once with $2 \times \mathrm{SSC}$, $0.1 \%$ SDS for $30 \mathrm{~min}$ at $65^{\circ} \mathrm{C}$; and finally with $0.2 \times \mathrm{SSC}$, $0.1 \%$ SDS for $30 \mathrm{~min}$ at $65^{\circ} \mathrm{C}$. Subsequently, the membranes were exposed to radiographic film with an intensifying screen at $-70{ }^{\circ} \mathrm{C}$. The membranes were stripped and rehybridized with a ${ }^{32} \mathrm{P}$-labelled human $\beta$-actin cDNA probe as a control of RNA gel loading. Densitometric analysis of hybridization signals was performed by the SCION IMAGE $1.62 \mathrm{c}$ program.

\subsection{Serial analysis of gene expression (SAGE)}

Virtual Northern analysis was carried out for all mt genes by accessing SAGEmap (NCBI web site http://www ncbi.nlm.nih.gov/SAGE). Five GB SAGE libraries (GSM696: SAGE_Duke_GBM_ H1110; GSM765: SAGE_pooled_GBM; GSM14767: SAGE_Brain_glioblastoma_B_H833; GSM 14768: SAGE_Brain_glioblastoma_B_R336; GSM14769: SAGE_Brain_glioblastoma_B_R70) and two normal human brain SAGE libraries (GSM676: SAGE_BB542_whitematter and GSM763: SAGE_ normal_pool(6th)) were selected for the displaying of the differences of $\mathrm{mt}$ genes expression.

\section{Results}

\subsection{Identification of genes with altered expression in $G B$ s by differential hybridization}

Hybridization of an arrayed human fetal brain cDNA library with total cDNA probes of GB and normal human adult brain revealed about one hundred cDNA clones with differences in the intensity of hybridization. Plasmid DNA from 80 cDNA clones received from RZPD was digested by HindIII/EcoRI restriction endonucleases, and after electrophoresis was blotted and hybridized with the same cDNA probes. Repeated differential hybridization of selected cDNA clones confirmed the results of the primary screening for 38 clones. Differential hybridization of one such plasmid DNA blot is shown in Fig. 1 where is possible to see that some cDNA inserts (lanes 10, 26) hybridized more intensive with a GB cDNA probe while the majority (lanes 1,5,9,15,16,19,24,25,28) hybridized more intensive with the normal adult human brain cDNA probe.

Nucleotide sequence analysis of cDNA inserts from 38 clones revealed 8 clones containing Alurepeats and 30 clones with cDNAs corresponding to mitochondrial genes ATP6, COXI, COXII, COXIII, ND1, ND4, and 12S rRNA (Table 1).

As it was expected in accordance with the results of primary screening, Alu-containing sequences

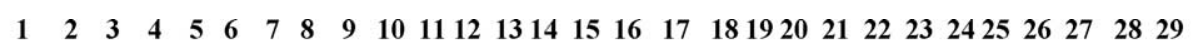

A

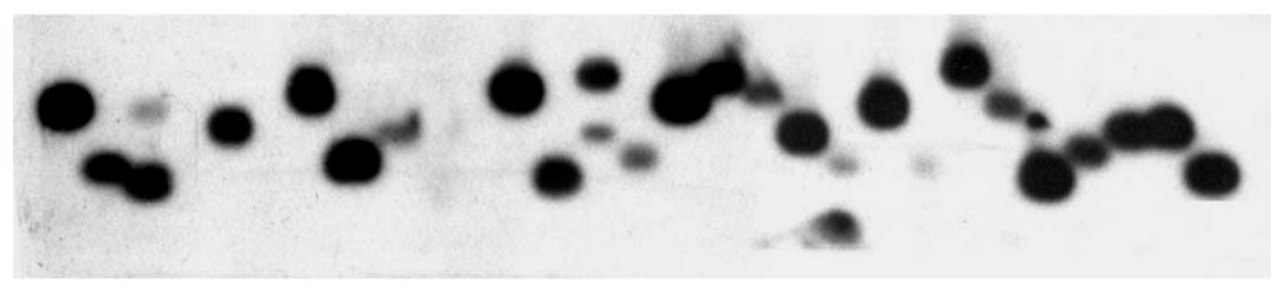

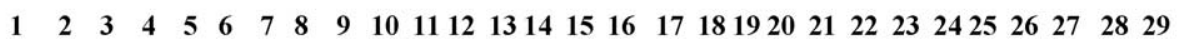

B

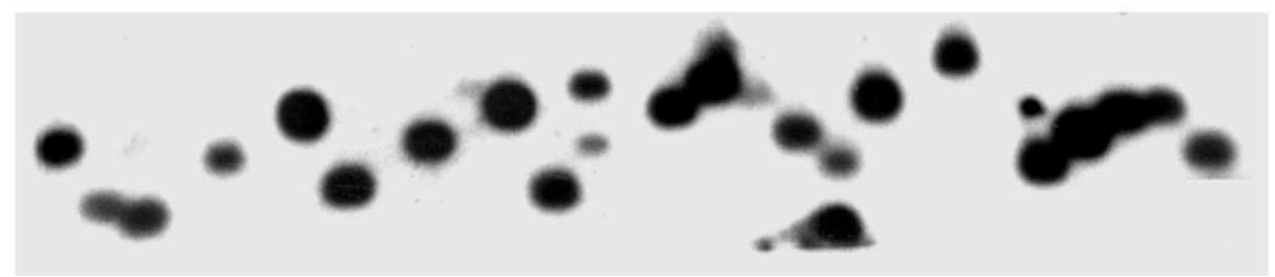

Fig. 1. Differential hybridization of plasmid DNAs with total cDNA probes of human normal brain (A) and GB (B). 
Table 1

Nucleotide sequences from human fetal brain (25 weeks old) cDNA library differentially expressed between glioblastoma and human normal brain

\begin{tabular}{|c|c|c|}
\hline Clone & $\begin{array}{l}\text { Expression changes } \\
\text { in glioblastoma }\end{array}$ & Gene name \\
\hline DKFZp415A2296 & decreased & mt NADH dehydrogenase subunit 1 \\
\hline DKFZp415D11116 & decreased & mt NADH dehydrogenase subunit 1 \\
\hline DKFZp415J1098 & decreased & mt NADH dehydrogenase subunit 1 \\
\hline DKFZp415L2318 & decreased & mt NADH dehydrogenase subunit 1 \\
\hline DKFZp415N0897 & decreased & mt NADH dehydrogenase subunit 1 \\
\hline DKFZp415N1579 & decreased & mt NADH dehydrogenase subunit 1 \\
\hline DKFZp415?1316 & decreased & mt NADH dehydrogenase subunit 4 \\
\hline DKFZp415G18136 & decreased & mt NADH dehydrogenase subunit 4 \\
\hline DKFZp415M02136 & decreased & mt NADH dehydrogenase subunit 4 \\
\hline DKFZp415P2074 & decreased & mt cytochrome oxidase subunit I \\
\hline DKFZp415G06136 & decreased & mt cytochrome oxidase subunit II \\
\hline DKFZp415E117 & decreased & mt cytochrome oxidase subunit III \\
\hline DKFZp415E1874 & decreased & mt cytochrome oxidase subunit III \\
\hline DKFZp415E1386 & decreased & mt cytochrome oxidase subunit III \\
\hline DKFZp415I1555 & decreased & mt cytochrome oxidase subunit III \\
\hline DKFZp415J19138 & decreased & mt cytochrome oxidase subunit III \\
\hline DKFZp415K05112 & decreased & mt cytochrome oxidase subunit III \\
\hline DKFZp415K1124 & decreased & mt cytochrome oxidase subunit III \\
\hline DKFZp415L07128 & decreased & mt cytochrome oxidase subunit III \\
\hline DKFZp415 & decreased & mt cytochrome oxidase subunit III \\
\hline N2116DKFZp415P2330 & decreased & mt cytochrome oxidase subunit III \\
\hline DKFZp415H0983 & decreased & mt ATP synthase subunit 6 \\
\hline DKFZp415J0588 & decreased & mt ATP synthase subunit 6 \\
\hline DKFZp415H20103 & decreased & mt ATP synthase subunit 6 \\
\hline DKFZp415I0891 & decreased & mt ATP synthase subunit 6 \\
\hline DKFZp415K0676 & decreased & mt ATP synthase subunit 6 \\
\hline DKFZp415L24135 & decreased & mt ATP synthase subunit 6 \\
\hline DKFZp415N0519 & decreased & mt ATP synthase subunit 6 \\
\hline DKFZp415N0413 & decreased & mt ATP synthase subunit 6 \\
\hline DKFZp415E0479 & decreased & mt $12 \mathrm{~S}$ rRNA \\
\hline
\end{tabular}

hybridized more intensively with glioblastoma total cDNA.

\subsection{Northern analysis of $m t$ genes expression in astrocytic gliomas}

Regardless of the type or combination of data for genes with altered expression in glioblastoma versus normal brain, there is still a need to confirm and expand the expression information Northern blot hybridization seems to be the best approach for this purpose. Indeed, Northern analysis of astrocytic gliomas of different malignancy grades showed the significantly lower level of ATP6, COXIII, COXII and ND1 mRNAs in GB (sample 182) as compared to normal brain (sample 181) of the same patient
(Fig. 2). These tissue samples were used for primary screening of the human fetal brain cDNA library. Mean values of ATP6, COXIII, COXII or ND1 mRNA content in GBs was about two fold lower than in normal brain. It varied greatly in different GB samples analyzed that likely reflected the molecular heterogeneity of these tumors.

The comparison of the pairs of astrocytic tumors and corresponding adjacent tissues of normal brain showed the difference in the mt mRNAs level for anaplastic astrocytoma (Fig. 2A-D, lanes 21 and 22) and even for astrocytoma grade II (Fig. 2A-D, lanes 19 and 20).

Northern hybridization with COXI (Fig. 3) and with ND4-probes (Fig. 4) showed similar patterns of the expression in normal brain and astrocytic tumors. 


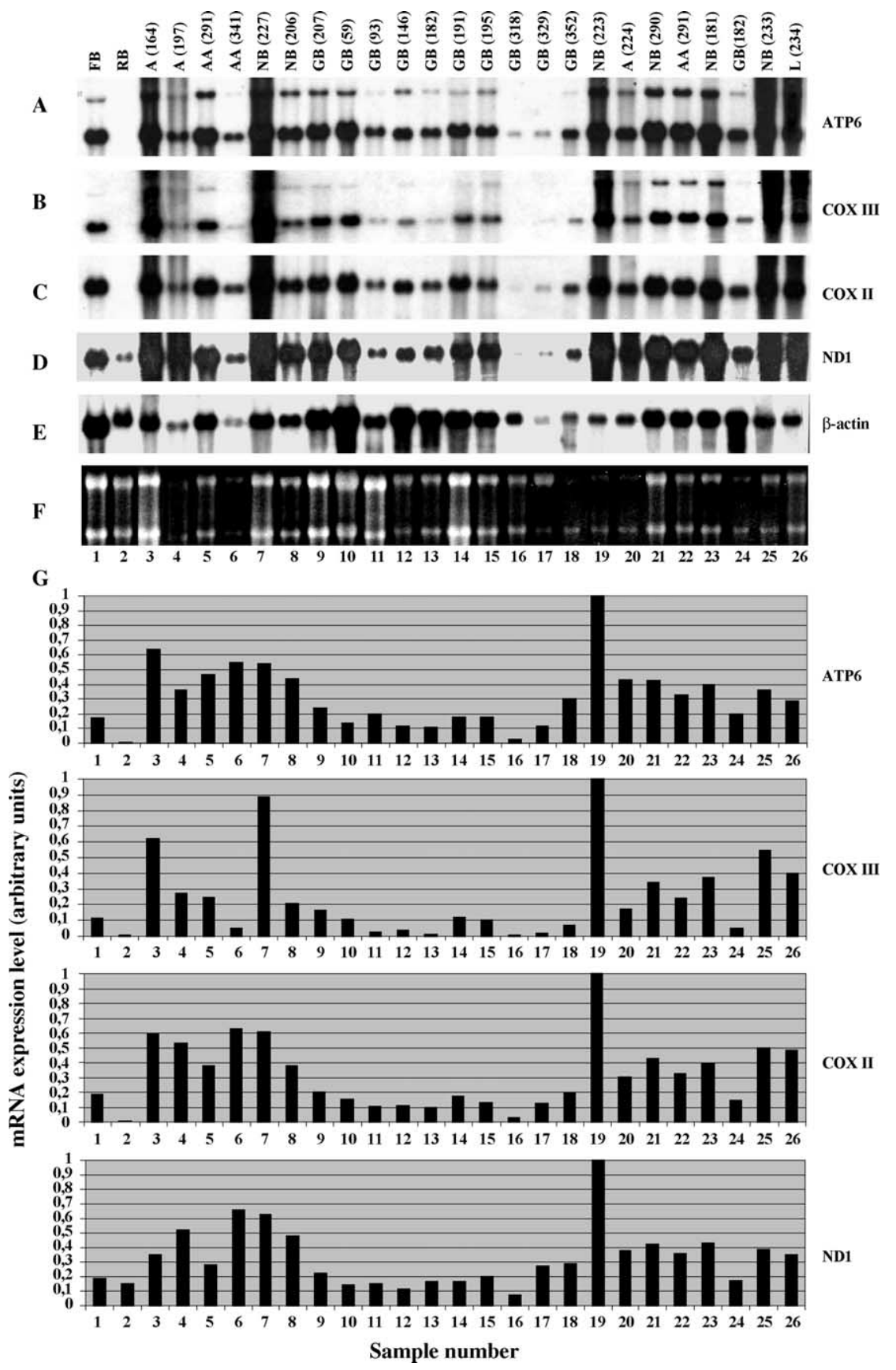

Fig. 2. Expression of ATP6, COX III, COXII and ND1 genes in normal human tissues and in brain tumors. Northern blot hybridization of ${ }^{32}$ P-labeled ATP6 (A), COXIII (B), COXII (C) and ND1 (D) cDNA probes with tumor RNA panel. Tissue types and tumor subtypes are indicated above each lane of the blot: FB, human fetal brain; RB, rat brain; NB, human normal brain; GB, glioblastoma; AA, anaplastic astrocytoma; A, astrocytoma; L, lymphoma. (E) Northern blot hybridization of the same blot with $\beta$-actin cDNA control probe. (F) Ethidium bromide stained agarose gel. (G) Bar graphs showing relative expression of $\mathrm{mt}$ genes after correction for gel loading based on $\beta$-actin gene expression. 


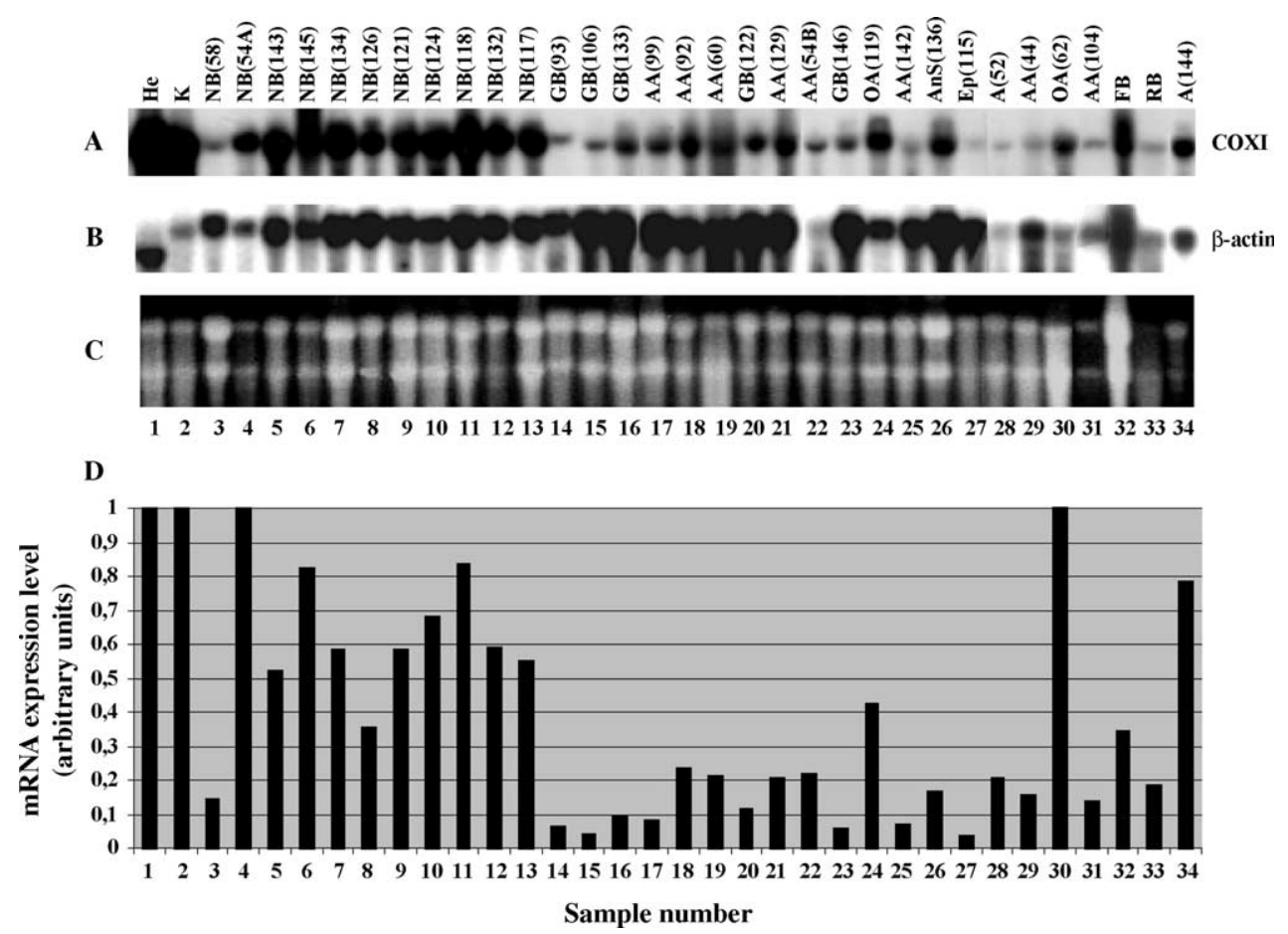

Fig. 3. Expression of COXI gene in normal human tissues and in brain tumors. (A) Northern blot hybridization of ${ }^{32} \mathrm{P}-\mathrm{labeled} \mathrm{COXI} \mathrm{cDNA}$ probe with tumor RNA panel. Tissue types and tumor subtypes are indicated above each lane of the blot: He, human heart; K, human kidney; $\mathrm{NB}$, human normal brain; GB, glioblastoma; AA, anaplastic astrocytoma; A, astrocytoma; OA, oligoastrocytoma; Ans, angiosarcoma; Ep, epidermoid; FB, human fetal brain; RB, rat brain. (B) Northern blot hybridization of the same blot with $\beta$-actin cDNA control probe. (C) Ethidium bromide stained agarose gel. (D) Bar graphs showing relative expression of COXI gene after correction for gel loading based on $\beta$-actin gene expression.

It is possible to see the decreased levels of these mRNAs in GB as compared to normal brain samples.

\subsection{Analysis of the expression of $m t$ genome in $G B$ and normal brain by $S A G E$}

Tag counts of all mt genes were examined in astrocytic gliomas and normal brain by accessing SAGEmap In the description to SAGE (http://www. ncbi.nlm.nih.gov/SAGE/) are some caviates that $\mathrm{mt}$ sequences were in large part masked or removed from Unigene but not from mapping of tags to mitochondrial sequences. A single NlaIII tag was revealed for each of the mt genes by utilizing the 'Retrieve by sequence' option, and expression of all $\mathrm{mt}$ genes in astrocytic gliomas was analyzed by 'Virtual Northern'. Thus, we obtained clear evidence that transcription levels of all mitochondrial genes are lower in glioblastoma than in the human normal brain (Table 2).

\section{Discussion}

Mitochondrial defects have long been suspected to play an important role in the development and progression of cancer. Several distinct differences between the mitochondria of cancer cells and normal cells have already been observed at the genetic, molecular and biochemical levels and these studies have indicated that mitochondrial dysfunction is involved in carcinogenesis. In particular, activation of the genes for COXI, COXII, and 16S rRNA was shown in rat fibroblast cells immortalized or transformed by viral and cellular oncogenes [14]; ND5, ND6, cytochrome $b$, COXII, and 16S rRNA in chemically induced rat hepatomas [15]; ND1, 12S 

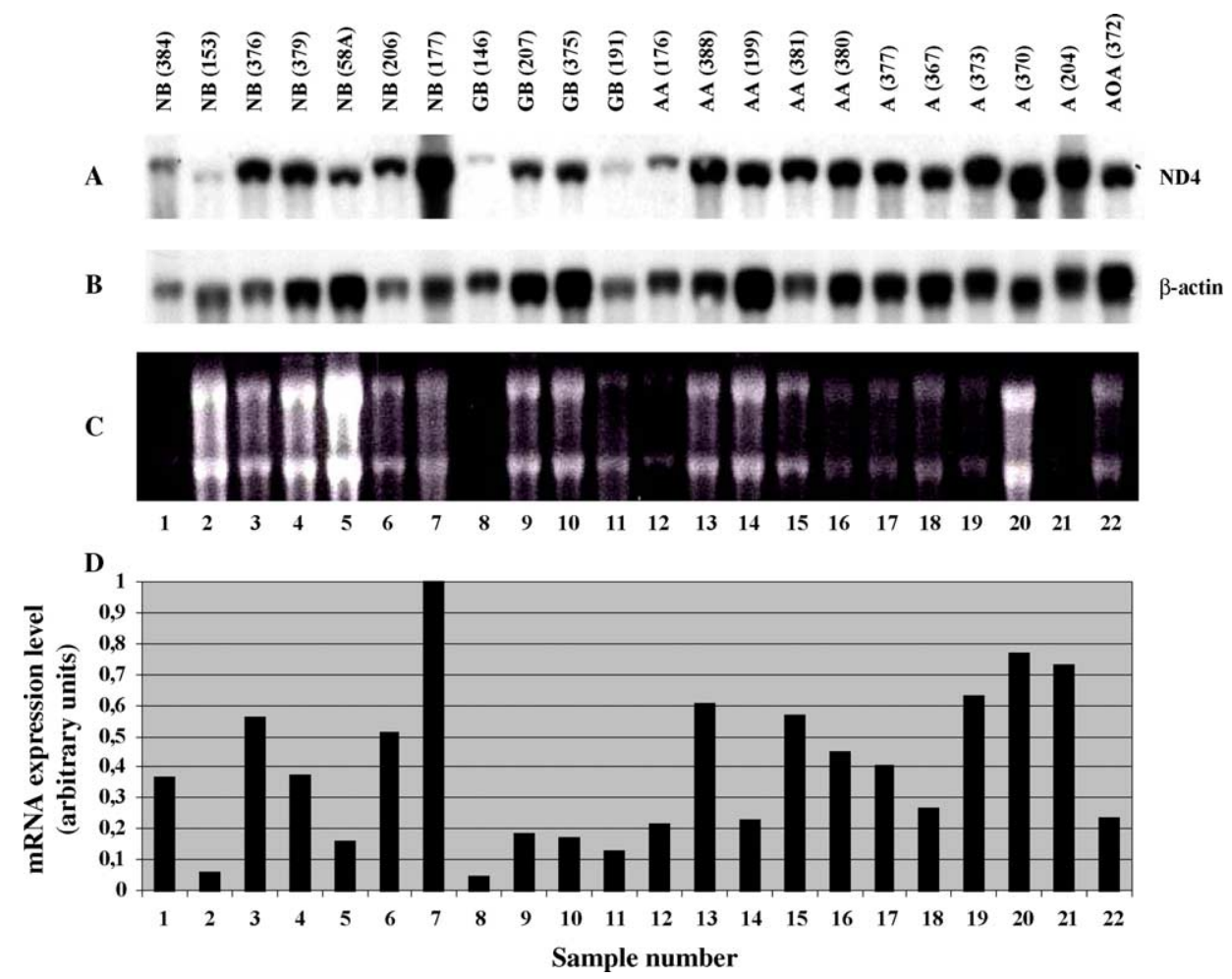

Fig. 4. Expression of ND4 gene in normal human tissues and in brain tumors. (A) Northern blot hybridization of ${ }^{32} \mathrm{P}-\mathrm{labeled}$ ND4 cDNA probe with tumor RNA panel. Tissue types and tumor subtypes are indicated above each lane of the blot: NB, human normal brain; GB, glioblastoma; AA, anaplastic astrocytoma; A, astrocytoma; AOA, anaplastic oligoastrocytoma. (B) Northern blot hybridization of the same blot with $\beta$-actin cDNA control probe. (C) Ethidium bromide stained agarose gel. (D) Bar graphs showing relative expression of ND4 gene after correction for gel loading based on $\beta$-actin gene expression.

rRNA, and 16S rRNA in polyps of familial polyposis coli patients [16]. Elevated levels of ATP6, ND5, cytochrome $b$, COXI, and COXII mRNAs were revealed in papillary thyroid carcinomas as compared to normal thyroid tissue [17]. Sharp et al. [18] detected activation of the COXII gene by analysis of 17 breast cancer tumors, but failed to detect the alterations in ATP6, ND2 and ND4 gene expression. Other mitochondrial enzyme levels (NADH cytochrome $c$ reductase, succinate cytochrome $c$ reductase, and cytochrome $c$ oxidase) have been shown to change also during exposure to carcinogens with cytochrome $c$ oxidase levels remaining elevated even after discontinuation of exposure [19]; increased levels of RNA transcripts of the nuclear-encoded subunit COXIV and mitochondrially encoded COX subunits I and II have been observed in Zajdela hepatoma as compared with normal liver [20]. A study utilizing tissues from 15 colorectal cancer patients found that ND2 mRNA levels were elevated in the malignant tissue as compared to normal tissue from the same individuals [21]. Analysis of the mitochondrial genome in colorectal cancer cell lines also revealed abnormalities; elevated expression of 16S rRNA, ND4, ND4L, cytochrome $b$, COXII, ATP6, and ATP8 was detected in the HT-29 cell line [22]. ND1, ND4, and COXII genes were shown to be overexpressed in B-cell non-Hodgkin's lymphomas in SIV-infected monkeys, 16S rRNA, COXII, ATP6, cytochrome $b$, ND2, and ND4-in human lymphomas [23].

In contrast to the investigations cited earlier, Zhou et al. [24] found that mitochondrial cytochrome $c$ oxidase (COX) activity was significantly diminished in $\mathrm{p} 53(-/-)$ cells. Their study suggests that p53 is involved in regulation of COXII at the protein level but not at the mRNA level. Decrease in COX activity was found also when comparing the specific activity 
Table 2

Results of 'Virtual Northern' of mt genes

\begin{tabular}{|c|c|c|c|c|c|c|c|c|}
\hline \multirow[t]{2}{*}{ Tag } & \multicolumn{7}{|c|}{ Tags per million } & \multirow[t]{2}{*}{ Gene name } \\
\hline & $\begin{array}{l}\text { NB A } \\
(94876)^{*}\end{array}$ & $\begin{array}{l}\text { NB B } \\
(63208)^{*}\end{array}$ & $\begin{array}{l}\text { GB C } \\
(70087)^{*}\end{array}$ & $\begin{array}{l}\text { GB D } \\
(61886)^{*}\end{array}$ & $\begin{array}{l}\text { GB E } \\
(100600)^{*}\end{array}$ & $\begin{array}{l}\text { GB F } \\
(102322)^{*}\end{array}$ & $\begin{array}{l}\text { GB G } \\
(99099)^{*}\end{array}$ & \\
\hline GTAAGTGTAC & 664 & 727 & 28 & 145 & 238 & 820 & 151 & $12 \mathrm{~S}$ rRNA \\
\hline CTAAGACTTC & 16864 & 10489 & 1983 & 3651 & 2773 & 3156 & 3239 & 16S rRNA \\
\hline CACCTAATTG & 9053 & 11739 & 2282 & 4314 & 2554 & 7153 & 15600 & ATP6/ ATP8 \\
\hline САСТАСТСАС & 1443 & 1439 & 570 & 646 & 0 & 1915 & 2875 & Cytochrome $b$ \\
\hline ATTTGAGAAG & 4163 & 6992 & 2297 & 2165 & 556 & 2804 & 1261 & COXI \\
\hline CCCATCGTCC & 11267 & 14001 & 4879 & 10616 & 4701 & 3107 & 17810 & COXII \\
\hline TGATTTCACT & 4279 & 4176 & 1383 & 1276 & 735 & 2413 & 5328 & COXIII \\
\hline ACCCTTGGCC & 6840 & 8748 & 870 & 1842 & 79 & 1847 & 2260 & ND1 \\
\hline ACTAACACCC & 6861 & 5505 & 784 & 1939 & 864 & 2609 & 2230 & ND2 \\
\hline AGCCCTACAA & 8621 & 8574 & 1169 & 3571 & 2713 & 5404 & 6518 & ND3 \\
\hline TTCATACACC & 5354 & 4002 & 3167 & 3183 & 7514 & 2756 & 14964 & ND4/ ND4L \\
\hline AGACCCACAA & 653 & 648 & 99 & 226 & 188 & 263 & 988 & ND5 \\
\hline GGGGTCAGGG & 242 & 221 & 57 & 145 & 79 & 107 & 191 & ND6 \\
\hline
\end{tabular}

SAGE libraries (total tag counts in library indicated in parentheses): A*, GSM676: SAGE_BB542_white matter; B*, GSM763: SAGE_normal pool (6th); C*, GSM696: SAGE_Duke_GBM_H1110; D*, GSM765: SAGE_pooled_GBM; E*, GSM14767: SAGE_Brain_glioblastoma_B_H833; F*, GSM 14768: SAGE_Brain_glioblastoma_B_R336; G*, GSM14769: SAGE_Brain_glioblastoma_B_R70.

of the enzyme in biopsies of human colonic adenocarcinoma versus normal colon mucosa [25], in cultured rat HC252 hepatoma cells versus nonneoplastic liver [26], and in cultured carcinoma cell lines MCF-7 (breast), T47D (breast) and DU-145 (prostate) versus normal epithelial cells [27]. FaureVigny et al. [28] detected the lower expression of several mitochondrial genes encoding the proteins involved in energy metabolism of cancer cells in renal tumors of different types: a clear cell carcinoma, an oncocytoma, and urothelial tumors. The mean level of expression of COX subunit III gene was found to be lower in carcinoma versus normal mucosa samples [29]. A separate study reported the loss of mtDNA and mRNA coding for subunit the ND3 gene in renal cell carcinoma [30].

Our results obtained by differential hybridization of arrayed human fetal brain cDNA library with GB and normal adult brain cDNA probes with subsequent sequence analysis of differentially hybridized cDNAs, Northern blot hybridization, and SAGE, revealed decreased expression of all mitochondrial genes in glioblastoma as compared to human normal adult brain. These results suppose possible coordinated changes of mitochondrial genes expression in GB. The reason of the decrease of mt mRNA levels is not known yet; it may be a consequence of the decrease of $\mathrm{mt}$ genome transcription activity, the increase of large mutations in mitochondrial DNA, or/and the decrease of quantity of mitochondria in glioblastomas. Discrepancies in the results concerning increase or decrease of the expression of $\mathrm{mt}$ genes in different tumors may be due to the different nature of various cancer types under investigation, and the reduction of all $\mathrm{mt}$ mRNA levels in GB may reflect the peculiarities of glioblastoma, the last grade of astrocytic glioma progression.

\section{Acknowledgements}

This work was supported in part by NATO Collaborative Linkage Grant No. 977284, grant No. F7/282-2001 (Fundamental Investigation Fond of Ukrainian Ministry of Education and Science), and by National Academy of Sciences of Ukraine in frames of the program 'Novel Problems of Medicine and Biology and Human Environment'.

\section{References}

[1] S. Weinhouse, Oxidative metabolism of neoplastic tissues, Adv. Cancer Res. 3 (1955) 269-325.

[2] P.L. Pedersen, Tumor mitochondria and the bioenergetics of cancer cells, Prog. Exp. Tumor Res. 22 (1978) 190-274. 
[3] E. Carafoli, Mitochondria and disease, Mol. Aspects Med. 3 (1980) 295-429.

[4] S. Ohta, A multi-functional organelle mitochondrion is involved in cell death, proliferation and disease, Curr, Med. Chem. 10 (2003) 2485-2494.

[5] J.B. Jones, J.J. Song, P.M. Hempen, G. Parmigiani, R.H. Hruban, S.E. Kern, Detection of mitochondrial DNA mutations in pancreatic cancer offers a 'mass'-ive advantage over detection of nuclear DNA mutations, Cancer Res. 61 (2001) 1299-1304.

[6] J.S. Penta, F.M. Johnson, J.T. Wachsman, W.C. Copeland, Mitochondrial DNA in human malignancy, Mutat. Res. 488 (2001) 119-133.

[7] J.S. Modica-Napolitano, K. Singh, Mitochondria as targets for detection and treatment of cancer, Expert Rev. Mol. Med. 2002; 1-19.

[8] J.S. Carew, P. Huang, Mitochondrial defects in cancer, Mol. Cancer 1 (2002) 1-9.

[9] W.C. Copeland, J.T. Wachsman, F.M. Johnson, J.S. Penta, Mitochondrial DNA alterations in cancer, Cancer Invest. 20 (2002) 557-569.

[10] P. Kleihues, D.N. Louis, B.W. Scheithauer, L.B. Rorke, G. Reifenberger, P.C. Burger, W.K. Cavenee, The WHO classification of tumors of the nervous system, J. Neuropathol. Exp. Neurol. 61 (2002) 215-225.

[11] T. Sambrook, E.F. Fritsch, T. Maniatis, Molecular cloning: a laboratory manual, second ed., Cold Spring Harbor Laboratory Press, Cold Spring Harbor, NY, 1989.

[12] K.O. Shostak, V.V. Dmitrenko, O.M. Garifulin, V.D. Rozumenko, O.V. Homenko, Y.P. Zozulya, G. Zehetner, V.M. Kavsan, Downregulation of putative tumor suppressor gene TSC-22 in human brain tumors, J. Surg. Oncol. 82 (2003) 57-64.

[13] P. Chomczynski, N. Sacchi, Single step method of RNA isolation by acid guanidinium thiocyanate-phenol-chloroform extraction, Anal. Biochem. 162 (1987) 156-159.

[14] N. Glaichennhaus, P. Leopold, F. Cuzin, Increased levels of mitochondrial gene expression in rat fibroblast cells immortalized or transformed by viral and cellular oncogenes, Eur. Mol. Biol. Org. J. 25 (1986) 1261-1265.

[15] M. Corral, B. Paris, G. Baffet, L. Tichonicky, C. GuguenGuillouzo, J. Kruh, N. Defer, Increased level of the mitochondrial ND5 transcript in chemically induced rat hepatomas, Exp. Cell Res. 184 (1989) 158-166.

[16] A. Yamamoto, S. Horai, Y. Yuasa, Increased level of mitochondrial gene expression in polyps of familial polyposis coli patients, Biochem. Biophys. Res. Commun. 2159 (1989) $1100-1106$.

[17] D.R. Haugen, O. Fluge, L.J. Reigstad, J.E. Varhaug, J.R. Lillehaug, Increased expression of genes encoding mitochondrial proteins in papillary thyroid carcinomas, Thyroid 13 (2003) 613-620.
[18] M.G.F. Sharp, S.M. Adams, R.A. Walker, Differential expression of the mitochondrial gene cytochrome oxidase II in benign and malignant breast cancer, J. Pathol. 168 (1992) 163-168.

[19] C.N. Huang, J.L. Tsai, M.T. Chen, W.J. Wu, K.W. Kuo, C.H. Huang, Changes in the activities of mitochondrial enzymes in the progress of tumorigenesis of bladder cancer, Biochem. Mol. Biol. Int. 46 (1998) 375-383.

[20] K. Luciakova, S. Kuzela, Increased steady-state levels of several mitochondrial and nuclear gene transcripts in rat hepatoma with a low content of mitochondria, Eur. J. Biochem. 205 (1992) 1187-1193.

[21] K.A. Chester, L. Robson, R.H. Begent, H. Pringle, L. Primrose, I.C. Talbot, A.J. Macpherson, S.L. Owen, G. Boxer, A.D. Malcolm, In situ and slot hybridization analysis of RNA in colorectal tumours and normal colon shows distinct distributions of mitochondrial sequences, J. Pathol. 162 (1990) 309-315.

[22] X. Lu, T. Walker, J.P. MacManus, V.L. Seligy, Differentiation of HT-29 human colonic adenocarcinoma cells correlates with increased expression of mitochondrial RNA: effects of trehalose on cell growth and maturation, Cancer Res. 52 (1992) 3718-3725.

[23] V.Z. Tarantul, G. Hunsmann, Mitochondrial polypeptides of the oxidative phosphorylation pathway as potential new targets for anti-cancer therapy, Med. Hypotheses 56 (2001) 386-387.

[24] S. Zhou, S. Kachhap, K.K. Singh, Mitochondrial impairment in p53-deficient human cancer cells, Mutagenesis 18 (2003) 287-292.

[25] A.S. Sun, K. Sepkowitz, S.A. Geller, A study of some mitochondrial and peroxisomal enzymes in human colonic adenocarcinoma, Lab. Invest. 44 (1981) 13-17.

[26] A.S. Sun, A.I. Cederbaum, Oxidoreductase activities in normal rat liver, tumor-bearing rat liver, and hepatoma HC-252, Cancer Res. 40 (1980) 4677-4681.

[27] J.S. Modica-Napolitano, S.E. Touma, Functional differences in mitochondrial enzymes from normal epithelial and carcinoma cells, in: Mitochondrial Dysfunction in Pathogenesis, Keystone Symposia, Silverthorne, 2000 p. 64.

[28] H. Faure-Vigny, A. Heddi, S. Giraud, D. Chautard, G. Stepien, Expression of oxidative phosphorylation genes in renal tumors and tumoral cell lines, Mol. Carcinog. 16 (1996) 165-172.

[29] B.G. Heerdt, H.K. Halsey, M. Lipkin, L.H. Augenlicht, Expression of mitochondrial cytochrome $c$ oxidase in human colonic cell differentiation, transformation, and risk for colonic cancer, Cancer Res. 50 (1990) 1596-1600.

[30] P. Selvanayagam, S. Rajaraman, Detection of mitochondrial genome depletion by a novel cDNA in renal cell carcinoma, Lab. Invest. 74 (1996) 592-599. 